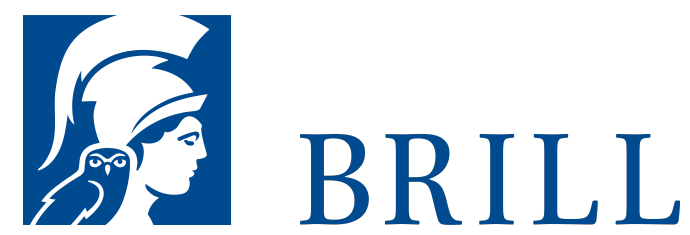

\title{
El espacio narrativo en la novela chilena postdictatorial
}

casas habitadas

Author: Bieke Willem

In El espacio narrativo en la novela chilena postdictatorial, Bieke Willem undertakes an analysis of the representation of space to identify a change in the poetics of recent fiction in Chile. Through a series of close readings of eight novels by some of Chile's most influential contemporary writers (Eltit, Marín, Bolaño, Fernández, Zambra and Zúñiga), she examines how Chilean authors in the period following the dictatorship deal in a melancholic or nostalgic way with the recent past and with changes in the meaning of space. The book combines reflections on literature, sociology, architecture and urbanism to paint a picture of what it means for these authors to be living in contemporary Chile.

En El espacio narrativo en la novela chilena postdictatorial, Bieke Willem define un cambio de paradigma poético en la narrativa chilena más reciente a partir de un análisis de la representación del espacio. A través del estudio detallado de ocho novelas escritas por los autores chilenos contemporáneos más influyentes (Eltit, Marín, Bolaño, Fernández, Zambra y Zúñiga), Willem examina cómo estos novelistas reelaboran el concepto del espacio después de la dictadura y se acercan de una manera melancólica o nostálgica al pasado reciente. El libro combina el estudio literario con otras disciplinas como la sociología, la arquitectura y el urbanismo para esbozar una imagen de qué significa...

See More

Readership

The book will appeal to anyone interested in the field of 21st century Hispanic and Latin American literature and culture in general and anyone concerned with the representation of space in contemporary literature.

A todos los interesados en la literatura y la cultura hispánica y

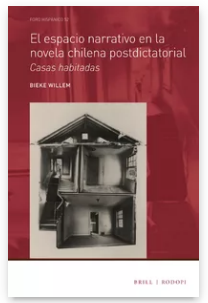

Pages: Approx.

475 pp.

Language:

Spanish

Subjects:

Hispanic

Studies,

Literature and

Cultural Studies, Criticism \&

Theory,

Literature and

Cultural Studies,

Social History,

History, Latin

America,

American

Studies

Publisher: Brill

Series:

Foro Hispánico,

Volume: $5^{2}$

E-Book (PDF)

Released online:

11 Jul 2016

ISBN: $978-90-$

04-3106o-5

List price

USD \$174.00

Hardback

Publication date:

28 Jul 2016 
latinoamericana y a aquellos lectores de estudios sobre la

representación del espacio en la literatura contemporánea.

ISBN: 978-90-

04-31057-5

List price

USD $\$ 174.00$ 
Bieke Willem, Ph.D., Ghent University, is a postdoctoral scholar at UC Berkeley. She has published on literary spatiality, autofiction and intimacy in contemporary Latin-American literature and film.

Bieke Willem, doctora, Universiteit Gent, es investigadora postdoctoral en UC Berkeley. Ha publicado sobre espacialidad literaria, autoficción e intimidad en el cine y la literatura latinoamericana contemporánea.

For more information see brill.com

\begin{abstract}
Order information: Order online at brill.com +44330 3330049 | customerservices@brill.com Submission information: brill.com/authors
\end{abstract}

Titles published by Brill | Fink, Brill | mentis or Brill | Schöningh: +49(o)71 5413279216 | brill@brocom.de 